\title{
Self-interference Channel for Full Duplex Transceivers
}

\author{
Alok Sethi \\ University of Oulu \\ Oulu, Finland \\ Email: asethi@ee.oulu.fi
}

\author{
Visa Tapio \\ University of Oulu \\ Oulu, Finland \\ Email:vita@ee.oulu.fi
}

\author{
Markku Juntti \\ University of Oulu \\ Oulu, Finland \\ Email: markku.juntti@ee.oulu.fi
}

\begin{abstract}
The self-interference (SI) channel in full duplex transceivers is investigated. The SI channel is measured using ultra wide-band antennas. Narrow-band measurement technique is used for the channel measurements so that spatial resolution of $4.3 \mathrm{~cm}$ is achieved. Measurements are done in a variety of locations including an anechoic chamber with different antenna orientation. Antennas are mounted on an old laptop frame. Coherence bandwidth of the SI channel is found to be varying between $1 \mathrm{MHz}$ and $4 \mathrm{MHz}$, effectively making it a frequency selective channel. It is also observed that a major amount of power is transferred because of direct coupling between the antennas via the frame on which antennas are mounted.
\end{abstract}

Keywords-Full duplex, Self-interference channel.

\section{INTRODUCTION}

Over the past few years, there has been an exponential growth in the mobile data sector [1]. To meet this growth, one of the focus area of the research community is full duplex (FD) transceivers. FD means simultaneous transmission and reception at the same frequency channel. For long, it was thought that it is not practically possible to simultaneously receive and transmit at the same frequency channel. In fact, given the difference in the power levels of the receive and transmit signal anyone can form a logical conclusion against the feasibility of the FD architecture. This prevalent understanding was, however, shattered by the work of Khandani [2] and Choi et al. [3]. The former unveiled an antenna architecture for the SI cancellation and the latter unveiled their first FD transceiver. The transceiver was a manually tuned system with a limited utility outside the lab environment. However, it was a vital proof-of-concept which proved the feasibility of the FD transceiver.

Taking a cue from the current FD transceiver implementations, a FD transceiver is going to have several cancellation stages with antenna cancellation stage being the first and the last being digital cancellation stage [3]-[8]. Since antenna cancellation is the first line of defense against the self transmitted signal, it is sensible to make it as effective as possible. For developing an effective cancellation scheme, one needs to know the channel between the transmit chain and the receive chain of the transceiver.

This paper investigates the self-interference (SI) channel using static measurements where the channel state is kept as constant as possible. A large number of measurements with a vector network analyzer (VNA) are taken at various locations in the frequency domain. The measured data is converted to time domain using inverse fast Fourier transform (IFFT). One of the key findings of this paper is the multipath nature of the SI channel. In particular cases, these multiple paths can have higher power compared to the line of sight (LOS) path. This behavior necessitates the need of an adaptive cancellation techniques which can not only cancel the LOS path but also the delayed version of the same.

This paper is organized in the following way: section II gives a brief overview of the system model and the challenges associated with the FD transceiver. It also discusses the important channel parameters. Section III describes the measurement setup and the plan used for channel measurements. Results are reported and discussed in Section IV. The paper is summarized in Section V.

\section{System Model}

SI is defined as the interference caused to the receiver by its own transmitted signal. Figure 1 shows a point-topoint communication system incorporating two nodes, clearly indicating the desired and SI signal paths. Let $P_{\mathrm{d}, i}$ and $h_{\mathrm{d}, i}$ be the power and the normalized channel for the desired signal received by the $i$ th node. Similarly, let $P_{\mathrm{i}, i}$ and $h_{\mathrm{i}, i}$ be the power and the normalized channel for the SI signal, as seen by the receiver of the $i$ th node. A valid assumption of symmetric uplink and downlink channel and equal transmitted power leads to a straightforward notation where $P_{\mathrm{i}}$ and $h_{\mathrm{i}}$ represent the power and normalized channel for the SI and $P_{\mathrm{d}}$ and $h_{\mathrm{d}}$ represent similar quantities for the desired channel respectively. For a receiver at any one of the nodes, let $X_{\mathrm{i}}$ and $X_{\mathrm{d}}$ be the data symbol for the SI and desired signal. Using a simple additive model, shown in Figure 2, input $Y_{i}(t)$ to a receiver of $i$ th node at time instant $t$ can be written as

$$
Y_{i}(t)=\sqrt{P_{\mathrm{i}}}\left(h_{\mathrm{i}}(t) * X_{\mathrm{i}}(t)\right)+\sqrt{P_{\mathrm{d}}}\left(h_{\mathrm{d}}(t) * X_{\mathrm{d}}(t)\right)+w(t),
$$

where white Gaussian noise is represented by $w$ and convolution operation is denoted by $*$. As seen from (1), the received signal is a combination of the desired and SI symbol. Since the receiver is only interested in the desired symbol, it needs a knowledge of the normalized SI channel before it can cancel out the effect of SI signal and estimate the desired symbol. As an example of a very simple SI cancellation mechanism, an inverted copy of the regenerated SI signal can be added at the 


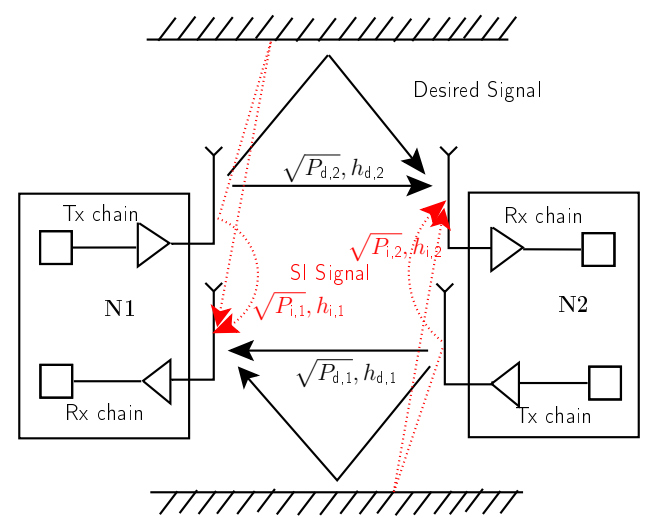

Fig. 1. System model.

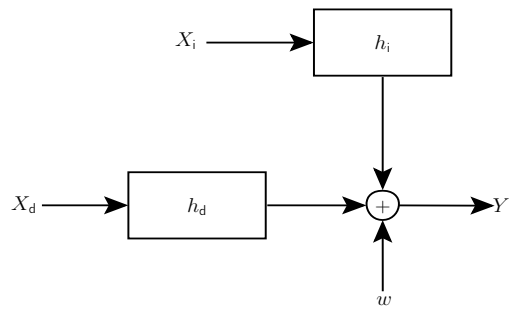

Fig. 2. Simplified system model.

receiver chain. This cancellation mechanism is illustrated as

$Y(t)=\sqrt{P_{\mathrm{i}}}\left(h_{\mathrm{i}}(t)-\hat{h}_{\mathrm{i}}(t)\right) * X_{\mathrm{i}}(t)+\sqrt{P_{\mathrm{d}}}\left(h_{\mathrm{d}}(t) * X_{\mathrm{d}}(t)\right)+w(t)$,

where, $\hat{h}_{\mathrm{i}}(t)$ represents an estimate of the SI channel at the receiver end. It can be also observed from (2) that the residual SI is directly related to the accuracy of the SI channel estimate, thus illustrating the importance of SI channel estimate.

In a typical digital transceiver, various functions like modulation, demodulation, frequency estimation, timing estimation, detection, etc., are done in the digital domain [9]. Wireless analog signals are converted to digital domain using an analogto-digital converter (ADC). ADCs have a limited dynamic range and is given by [10]

$$
D R=6.021 N_{\mathrm{ADC}}+1.763+10 \log _{10} \frac{f_{\mathrm{s}}}{2 B} \mathrm{~dB} .
$$

Here $N_{\mathrm{ADC}}$ is the number of bits in the $\mathrm{ADC}, f_{\mathrm{s}}$ is the sampling frequency and $B$ is the bandwidth of the signal of interest. The term $10 \log _{10} \frac{f_{\mathrm{s}}}{2 B}$ represents the processing gain that is achieved by oversampling. In wireless local area network (WLAN) systems, the power of the transmitted signal is of the order of $20 \mathrm{dBm}$ and of the desired received signal is around $-70 \mathrm{dBm}$. Assuming that an analog radio frequency (RF) cancellation scheme can cancel out about $50 \mathrm{~dB}$ of SI, the power of the SI signal at the input of the ADC will be $-30 \mathrm{dBm}$. For a $20 \mathrm{MHz}$ wide channel, value of thermal noise power at room temperature comes out to be $-100 \mathrm{dBm}$. Now, considering these figures and normalized channel gains of unity in (1), (3) yields that at least 12 bit ADC is required. This ADC bit requirement is 7 bits more if there was no SI signal. This sample calculation gives a rough idea of the importance of the SI cancellation before the digital domain.
The important channel parameters on which this work focuses are isolation between antennas, root mean square (RMS) delay $\left(\tau_{\text {RMS }}\right)$ and coherence bandwidth $\left(B_{\mathrm{c}}\right)$ of the SI channel. As the measurements are done in a static environment, Doppler spread of the channel is not investigated. The two-port network consisting of antennas and self-interference channel is assumed to be reciprocal i.e., $S_{21}$ is equal to $S_{12}$, thus, only $S_{21}$ parameter is collected during the measurement campaign. The frequency domain measurements are converted to time domain by applying the IFFT operation on the $S_{21}$ parameter thus yielding power delay profile (PDP). A PDP can be characterized by the following parameters [11]:

- excess delay,

- total excess delay,

- mean delay,

- RMS delay.

The excess delay is the delay of a path relative to LOS component delay. Total excess delay is the delay between the last significant (power above the noise floor) path and the LOS path. The mean delay, $\tau_{0}$, represents the first moment of the PDP. Mathematically, it is represented by the following equation

$$
\tau_{0}=\frac{\sum_{i=1}^{i=N_{\mathrm{n}}} P_{i} \tau_{i}}{\sum_{i=1}^{i=N_{\mathrm{n}}} P_{i}},
$$

where $P_{i}$ is the power of the $i$ th path and $\tau_{i}$ is the relative delay of the same. RMS delay, $\tau_{\text {RMS }}$, is the second moment of the PDP. It is given by

$$
\tau_{\mathrm{RMS}}=\sqrt{\frac{\sum_{i=1}^{i=N_{\mathrm{n}}} P_{i} \tau_{i}^{2}}{\sum_{i=1}^{i=N_{\mathrm{n}}} P_{i}}-\tau_{0}^{2} .}
$$

RMS delay is independent of actual path delays and is defined only by the relative delays, thus, making it a good indicator of the system delay spread. It is also related to the coherence bandwidth of the channel. $B_{\mathrm{c}}$ is the range of frequencies over which it can be assumed that the channel is experiencing flat fading. Assuming $B_{\mathrm{c}}$ is defined as a band over which frequency transfer function of the channel has a correlation factor of 0.9 , $B_{\mathrm{c}}$ is given as [12]

$$
B_{\mathrm{c}} \approx \frac{1}{50 \tau_{\mathrm{RMS}}} .
$$

Given the large power difference between the SI and desired signal, it is logical to have minimum variance in the SI channel estimate. As the value of $B_{\mathrm{c}}$ dictates the pilot structure which in turn dictates the variance in channel estimate, it is justified that a correlation factor of 0.9 is used to define $B_{\mathrm{c}}$.

\section{MeAsurement SetuP}

The measurement setup was designed keeping in mind a FD transceiver with a separate receive and transmit antenna. Agilent VNA 8720 ES [13] along with double loop antennas 


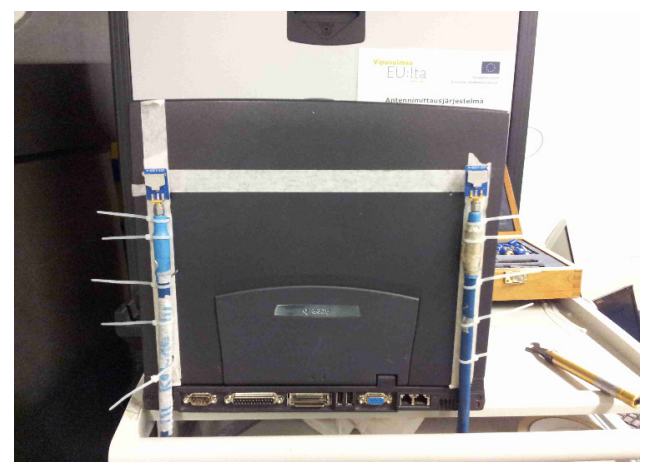

Fig. 3. Symmetrically aligned antennas.

from SkyCross, model number SMT-3TO10M-A [14] were used for this work. This particular antenna was chosen because of its compact size and ultra wide-band characteristics. The $S_{11}$ parameter of the antennas was measured over the whole band to ensure that the antennas are radiating over the whole frequency band. The antenna was a linearly polarized antenna with a maximum gain of $4.4 \mathrm{dBi}$ at $4.5 \mathrm{GHz}$. Phase stable cables, model number SUCOFLEX 104PEA from Huber+Suhner, were employed for connecting the antennas with the VNA. The antennas were mounted on an old laptop frame. Measurements were done in two separate campaigns. During the first campaign [8], three separate locations and four different antenna orientations were analyzed. During the second campaign, two antenna orientations and two different locations were analyzed. Antenna orientations varied from symmetric to all three planes orthogonal to each other. Figures 3-6 shows the different antenna orientations. Based on the results of campaign one, two antenna orientations were selected and more exhaustive measurements were done for those. During the second campaign, at each location a square grid was created with the distance between the neighboring points as $3 \mathrm{~cm}$. Overall dimensions of the grid was $15 \mathrm{~cm} \times 15 \mathrm{~cm}$ giving a total of 36 points. Separation distance of $3 \mathrm{~cm}$ was selected because that was the minimum wavelength of the electromagnetic wave used. The reason for creating a grid and to move the base unit in it is to average out any constructive or destructive interference from the environment.

During the first campaign, the locations were; an anechoic chamber of dimensions $11.5 \mathrm{~m} \times 6.5 \mathrm{~m} \times 6.5 \mathrm{~m}$, center of a lab with the following dimensions $5.5 \mathrm{~m} \times 6.5 \mathrm{~m} \times 4 \mathrm{~m}$ and inside the same lab with antennas facing a metal wall. The distance between the antennas and the wall was $30 \mathrm{cms}$. In the second campaign, measurements were done in an office of dimensions $4.16 \mathrm{~m} \times 2.89 \mathrm{~m} \times 3.27 \mathrm{~m}$ and in an office corridor as shown in Figure 7.

Few of the important VNA configuration parameters are listed in Table I. Before doing any measurements, VNA was calibrated using vector-error correction. Full two-port calibration was done as defined in the service manual of the VNA [15], [16]. After the calibration was finished, the base unit i.e., the laptop frame with antennas mounted on the back face, was placed at different locations. The base unit was seated on an electromagnetic absorber. Error corrected data i.e., the data after applying the calibration data, was captured on a computer using a LabView application. The computer

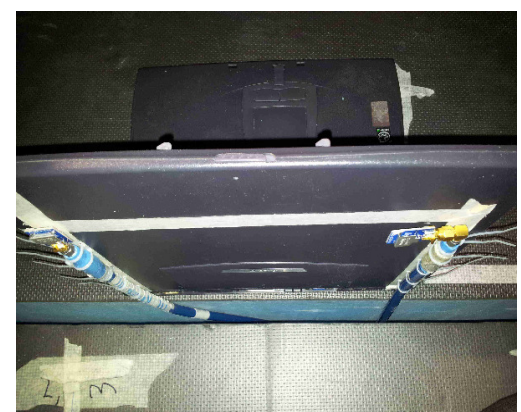

Fig. 4. Spatial arrangement of antennas with $\mathrm{XY}$ and $\mathrm{YZ}$ planes orthogonal.

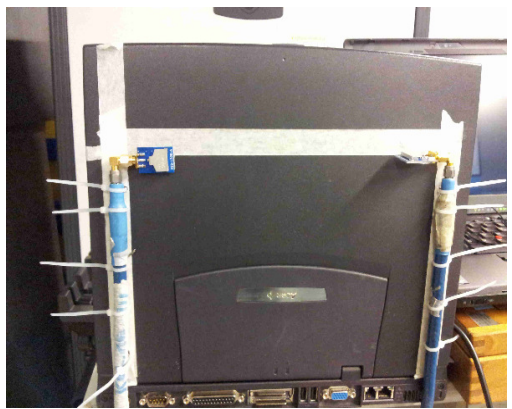

Fig. 5. Spatial arrangement of antennas with $\mathrm{YZ}$ and $\mathrm{XZ}$ planes orthogonal.

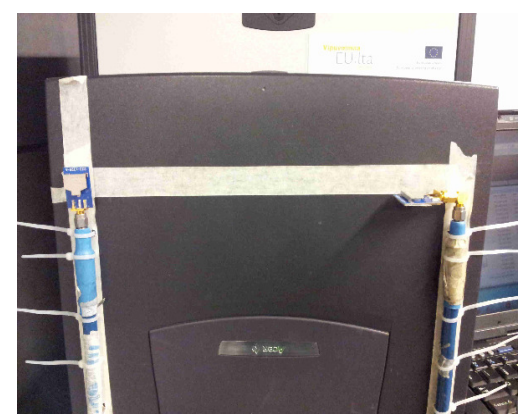

Fig. 6. Spatial arrangement of antennas with all three planes orthogonal to each other.

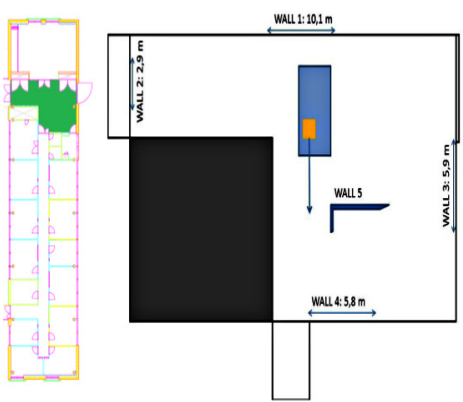

Fig. 7. Corridor layout.

was connected to the VNA via the general purpose interface bus (GPIB) interface [17]. For each measurement configuration 100 sweeps were collected which were then processed in Matlab. Number of sweeps was limited to 100 because it was found experimentally that the standard deviation of the data was constant with the increasing number of sweeps i.e., averaging more than 100 sweeps was not reducing the noise 
TABLE I. VNA PARAMETERS

\begin{tabular}{|c|c|}
\hline Parameter & Value \\
\hline Frequency band & 3 to $10 \mathrm{Ghz}$ \\
\hline Bandwidth $\left(B_{\mathrm{V}}\right)$ & $7 \mathrm{Ghz}$ \\
\hline Number of points $\left(N_{\mathrm{V}}\right)$ & 1601 \\
\hline Sweep time & 800 milli-seconds \\
\hline Transmitted power & $5 \mathrm{dBm}$ \\
\hline
\end{tabular}

in measurements. During the second measurement campaign, PDP for each grid point was calculated and then all the 36 PDPs were averaged out.

\section{RESULTS}

Table II shows the average value of isolation achieved with various antenna orientations at different locations. As expected, with all three planes orthogonal to each other, antenna orientation defined in Setup 4 achieves the maximum amount of isolation. It can be also seen from Table II that in the case where there are no external reflections,i.e., anechoic chamber, relative orientation of the antennas with respect to each other has a little impact on the amount of isolation. This suggests that the majority of power is transferred via the coupling between the antennas and frame. Furthermore, there is a drop in the average amount of isolation when there are strong reflectors nearby. Figure 8 shows the power collected under different time intervals, relative to the power of the lineof-sight component. As the distance between the antennas is less than $30 \mathrm{~cm}$, line-of-sight component should arrive under $1.5 \mathrm{~ns}$, even after considering the extra delay as suggested in [18]. As it can be seen from Figure 8 that even in the absence of an external reflector, the power collected in second bin of $1.5 \mathrm{~ns}$ to $3 \mathrm{~ns}$, is not zero. This implies there are secondary paths longer than the line-of-sight paths and they have power comparable to the line-of-sight path. Another thing to notice in Figure 8 is that different antenna orientations yield different relative power of the secondary component. One possible explanation for this behavior lies in the polarization of the antenna. As the antennas are linearly polarized and given their orthogonal relative orientation, it can be argued that electromagnetic waves reflected from the laptop structure are responsible for this behavior. Similar behavior was found for the lab environment as can be seen from Figure 9. While doing measurements inside the anechoic chamber and lab, the distance between the base unit and any potential reflector was more than a meter (except the third configuration where metal wall was about $35 \mathrm{~cm}$ from the base unit). Considering that and assuming speed of light to be $3 \times 10^{8} \mathrm{~m} / \mathrm{s}$, it can be deduced that any reflected component will take more than 3 ns. Thus, the secondary component shown in Figures 8 and 9 can only be due to the reflections from base unit itself. Figure 10 illustrates the power distribution in case there are metal reflectors nearby. Here, it can be seen that the power of the reflected components is much higher than the line-of-sight components. Figure 11 illustrates the same for the combined office and corridor environment. One thing to be noted about office environment is the relative small power of the secondary paths. Both office and corridor measurements were combined for plotting the collected power graphs as there was only a marginal difference in the collected powers for them. Table III illustrates the average value of coherence bandwidth calculated in various cases. $B_{\mathrm{c}}$ for an individual sweep was calculated by
TABLE II. AVERAGE ISOLATION (DB).

\begin{tabular}{|c|c|c|c|c|c|}
\cline { 2 - 6 } \multicolumn{1}{c|}{} & Anechoic chamber & Lab & Lab (reflector) & Office & Corridor \\
\hline Setup 1 & 38.49 & 40.63 & 34.40 & - & - \\
\hline Setup 2 & 40.40 & 41.05 & 40.38 & - & - \\
\hline Setup 3 & 39.87 & 41.07 & 35.14 & 41.9 & 41.8 \\
\hline Setup 4 & 41.76 & 43.44 & 38.85 & 44.9 & 46.5 \\
\hline
\end{tabular}

TABLE III. AVERAGE $B_{\mathrm{C}}$ (IN MEGA HERTZ).

\begin{tabular}{|c|c|c|c|c|c|}
\cline { 2 - 6 } \multicolumn{1}{c|}{} & Anechoic chamber & Lab & Lab (reflector) & Office & Corridor \\
\hline Setup 1 & 2.51 & 2.28 & 2.69 & - & - \\
\hline Setup 2 & 4.22 & 2.60 & 2.26 & - & - \\
\hline Setup 3 & 1.24 & 1.15 & 2.99 & 1.9 & 4.0 \\
\hline Setup 4 & 2.95 & 4.08 & 3.91 & 3.2 & 4.0 \\
\hline
\end{tabular}

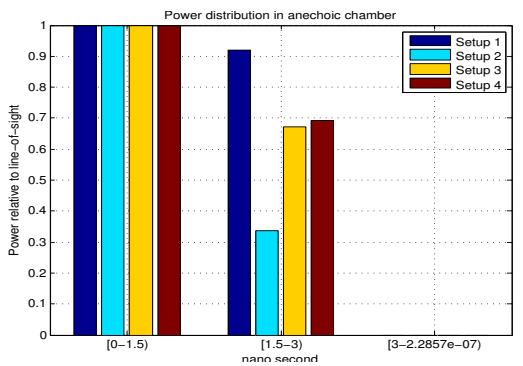

Fig. 8. Power distribution in anechoic chamber.

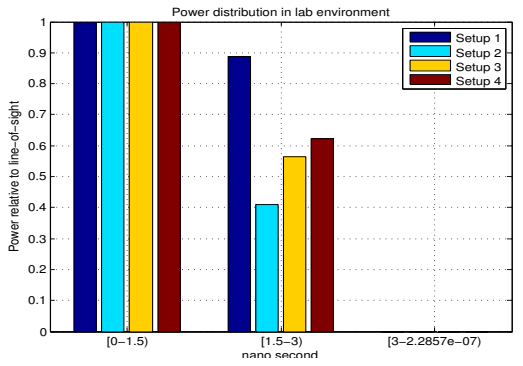

Fig. 9. Power distribution in lab environment.

applying (4), (5) and (6) on the corresponding PDP data. A low value of $B_{\mathrm{c}}$ indicates that power is distributed among multiple paths of different delays. It is interesting to note that under the corridor environment value of $B_{\mathrm{c}}$ is same for different antenna orientations.

Figure 12 shows the high degree of correlation (red color represents correlation value of 1) among the PDPs collected at different grid points in the corridor measurements. This suggests that there was no change in the PDP when the base unit was being moved i.e., there was no impact of the surroundings on the multiple paths of the SI channel. This behavior was not observed in the correlation data in the office environment as can be seen from Figure 13, where correlation was found to be decreasing with increasing separation distance.

\section{CONCLUSIONS}

The goal of this paper was to have a better understanding of the self-interference channel in full duplex communications. It is needed to develop robust cancellation mechanisms and realistic performance simulators. A narrow-band channel measurement technique was used. Using UWB antennas, the SI channel was measured. The results from the channel measurement exercise showed that the SI channel is a multi-path channel. It should be duly noted that here the delay between 


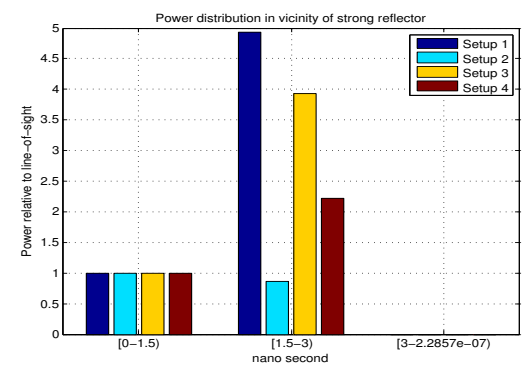

Fig. 10. Power distribution in proximity of strong reflector.

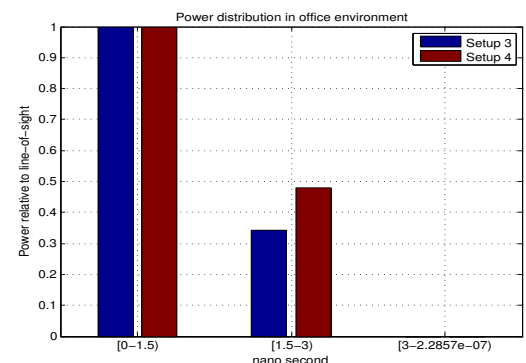

Fig. 11. Power distribution in office and corridor environment.

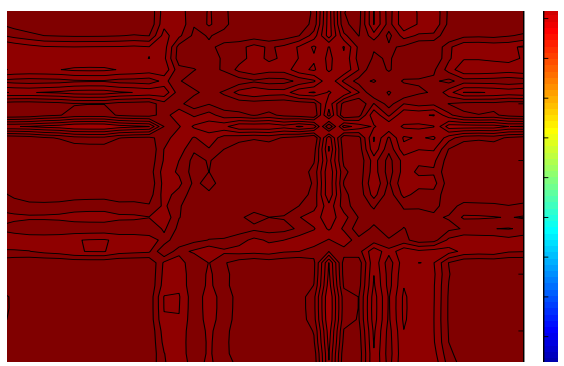

Fig. 12. Correlation among PDP of different grid points in corridor environment.

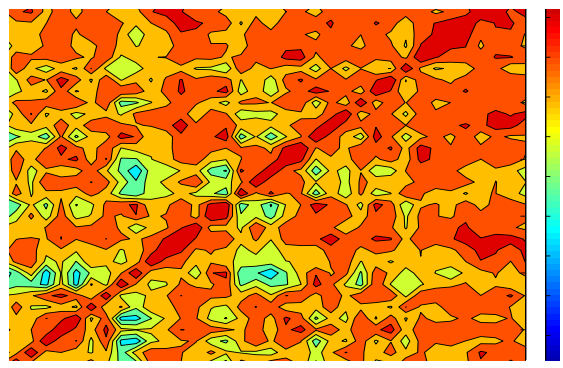

Fig. 13. Correlation among PDP of different grid points in office environment.

different paths is in the order of nano seconds as compared to micro or milli second order of the indoor wireless channels. It was also found that polarization based isolation does not work when the antennas are attached to a frame. The lowest value of $B_{\mathrm{c}}$ was found to be around $1 \mathrm{MHz}$. The measurements were done in an environment that closely resembled the general operating conditions of IEEE 802.11 devices.

The results presented in this work are from a work under progress. This work gives a preliminary overview of the SI channel. Currently available measurements are a fraction of those needed to develop a statistical model. As suggested during the review of this work also, measurements with antennas mounted on different surfaces, for example, a wall, will provide further insights and will be considered for future measurements.

\section{ACKNOWLEDGMENT}

The research leading to these results has received funding from the European Union Seventh Framework Programme (FP7/2007-2013) under grant agreement No. 316369 - project Full-Duplex Radios for Local Access (DUPLO). The authors would also like to thank Paavo Ollikainen for doing the required measurements.

\section{REFERENCES}

[1] "Cisco: Global mobile data traffic forecast," visited on 2013-6-19. [Online]. Available: http://www.cisco.com/en/US/solutions/collateral/ ns341/ns525/ns537/ns705/ns827/white_paper_c11-520862.pdf

[2] A. Khandani, "Methods for spatial multiplexing of wireless two-way channels," Patent USA 7817641, 10 19, 2010. [Online]. Available: http://www.google.com/patents/US7817641

[3] J. I. Choi, M. Jain, K. Srinivasan, P. Levis, and S. Katti, "Achieving single channel, full duplex wireless communication," in MobiCom, Chicago, Illinois, USA, Sep. 20-24 2010, pp. 1-12.

[4] M. Jain, J. I. Choi, T. Kim, D. Bharadia, S. Seth, K. Srinivasan, P. Levis, S. Katti, and P. Sinha, "Practical, real-time, full duplex wireless," in MobiCom. New York, NY, USA: ACM, 2011, pp. 301-312.

[5] M. Duarte and A. Sabharwal, "Full-duplex wireless communications using off-the-shelf radios: Feasibility and first results," in Asilomar Conference on Signals, Systems and Computers (ASILOMAR), nov 2010 , pp. $1558-1562$.

[6] A. Sahai, G. Patel, and A. Sabharwal, "Pushing the limits of full-duplex: Design and real-time implementation," CoRR, vol. abs/1107.0607, 2011.

[7] A. Khandani, "Two-way (true full-duplex) wireless," in 2013 13th Canadian Workshop on Information Theory (CWIT), 2013, pp. 33-38.

[8] A. Sethi, "Self-interference channel and analog baseband cancellation for full duplex transceiver," Master's thesis, University of Oulu, August 2013.

[9] H.Meyr, M.Moeneclaey, and S. Fechtel, Digital Communication Receivers, 1st ed. John Wiley \& Sons, Inc., 1997.

[10] T. C. Carusone, D. A. Johns, and K. Martin, Analog Integrated Circuit Design, 2nd ed. John Wiley \& Sons, Inc., 2011.

[11] S. R. Saunders and A. A. Zavala, Antennas and propagation for wireless communication systems, 2nd ed. John Wiley \& Sons, Inc., 2007.

[12] B. Sklar, "Rayleigh fading channels in mobile digital communication systems .i. characterization," IEEE Communications Magazine, vol. 35, no. 7, pp. 90-100, 1997.

[13] "Agilent vna," visited on 2013-6-11. [Online] Available: http://www.home.agilent.com/en/pd-1000002256\%3Aepsg\% 3 Apro-pn-8720ES/s-parameter-network-analyzer?\&cc=FI\&lc=fin

[14] "Skycross," visited on 2013-6-11. [Online]. Available: www.skycross $\mathrm{com} /$

[15] "8719et/es, 8720et/es, and 8722et/es network analyzers user's guide (june02)," visited on 2013-6-11. [Online]. Available: $\quad$ http://www.home.agilent.com/agilent/redirector.jspx? action=ref \&cname=AGILENT_EDITORIAL\&ckey $=1000000104-1 \%$ 3Aepsg\%3Aman\&lc=fin\&cc=FI\&nfr=-536902656.536881995.00

[16] "Agilent network analyzer basics," visited on 2013-6-11. [Online]. Available: http://cp.literature.agilent.com/litweb/pdf/5965-7917E.pdf

[17] V. Hovinen, M. Hamalainen, and T. Patsi, "Ultra wideband indoor radio channel models: preliminary results," in IEEE Conference on Ultra Wideband Systems and Technologies, 2002. Digest of Papers. 2002, 2002, pp. 75-79.

[18] T. Kumpuniemi, T. Tuovinen, M. Hamalainen, K. Yazdandoost, R. Vuohtoniemi, and J. Iinatti, "Measurement-based on-body path loss modelling for uwb wban communications," in International Symposium on Medical Information and Communication Technology (ISMICT), 2013, pp. 233-237. 